\title{
NORMS, KERNELS AND EIGENVALUES OF SOME INFINITE GRAPHS
}

\author{
Aahan Agrawal, Astrid Berge, Seth Colbert-Pollack, Rubén A. \\ MARTÍNEZ-AVENDAÑO AND ElySSA SliHEET
}

\begin{abstract}
In this paper we study the adjacency matrix of some infinite graphs, which we call the shift operator on the $L^{p}$ space of the graph. In particular, we establish norm estimates, we find the norm for some cases, we decide the triviality of the kernel of some infinite trees, and we find the eigenvalues of certain infinite graphs obtained by attaching an infinite tail to some finite graphs.
\end{abstract}

Mathematics subject classification (2010): 05C63, 05C50, 47A05, 47A10, 47A75.

Keywords and phrases: Infinite graphs, infinite trees, norm, kernel, spectrum, eigenvalues.

\section{REFERENCES}

[1] N. L. Biggs, Bojan Mohar and John Shawe-Taylor, The spectral radius of infinite graphs, Bull. London Math. Soc. 20 (1988) 116-120.

[2] Andries E. Brouwer and Willem H. Haemers, Spectra of Graphs, Universitext, Springer, 2012.

[3] Flavia Colonna And Rubén A. Martínez-Avendaño, Some classes of operators with symbol on the Lipschitz space of a tree, Mediterr. J. Math. 14 (2017) 1, Art. 18, 25.

[4] H. S. M. COXETER, Regular polytopes, Dover, 1973.

[5] Peter L. Duren, On the spectrum of a Toeplitz operator, Pacific J. Math. 14 (1964) 21-29.

[6] ZDENĚK DVOŘÁK AND BOJAN MOHAR, Spectral radius of finite and infinite planar graphs and of graphs of bounded genus, J. Combin. Theory Ser. B 100 (2010) 729-739.

[7] Roger A. Horn and Charles R. Johnson, Matrix Analysis, Cambridge University Press, 1985.

[8] L. Golins KII, Spectra of infinite graphs with tails, Linear Multilinear Algebra 64 (2016) 2270-2296.

[9] L. Golins KiI, Spectra of infinite graphs via Schur complement, Oper. Matrices 11 (2017) 389-396.

[10] L. GolinS KII, Spectra of comb graphs with tails, arXiv:1904.06678, 2019.

[11] V. O. LEBID' AND L. O. NYZHNYK, Spectral analysis of some graphs with infinite rays, (Translation of Ukraïn. Mat. Zh. 66 (2014), no. 9, 1193-1204) Ukrainian Math. J. 66 (2015) 1333-1345.

[12] L. P. NizHnik, Spectral analysis of metric graphs with infinite rays, Methods Funct. Anal. Topology 20 (2014) 391-396.

[13] Robert E. Megginson, An Introduction to Banach Space Theory, Springer, 1998.

[14] Bojan MOHaR, The spectrum of an infinite graph, Linear Algebra Appl. 48 (1982) 245-256.

[15] BoJAn Mohar, Some relations between analytic and geometric properties of infinite graphs, in Directions in infinite graph theory and combinatorics (Cambridge, 1989), Discrete Math. 95 (1991) 193219.

[16] Bojan Mohar And Wolfgang Woess, A survey on spectra of infinite graphs, Bull. London Math. Soc. 21 (1989) 209-234.

[17] Vladimir MÜLler, Spectral theory of linear operators and spectral systems in Banach algebras, Operator Theory: Advances and Applications 139, second edition, Birkhäuser Verlag, Basel, 2007.

[18] JOACHIM VON BELOW, An index theory for uniformly locally finite graphs, Linear Algebra Appl. 431 (2009) 1-19. 